\title{
In Silico Studies on Structural Function of Melanin Concentrating Hormone Receptor 1 Through Docking Approach, Towards Designing Drug for Treating Obesity
}

\author{
Mutangana Dieudonne*, Musafili Narcisse, Nyurahayo Jean Gaetan, and Munyampundu Jean Pierre \\ University of Rwanda, College of Science and Technology, Department of Biology, Avenue de l'Armée, \\ Po. Box 3900, Kigali-Rwanda \\ * Corresponding author: Mutangana Dieudonne, mature02@gmail.com
}

\begin{abstract}
Melanin concentrating hormone receptor 1 is a G-protein coupled protein receptor expressed in the lateral hypothalamus and zona incerta, part of the nervous system that regulates feeding behavior and energy homeostasis. It is involved in the stimulation of appetite, this was seen when synthetic MCHR1 or MCH was administered to mice and it resulted in induced obesity due to the enhanced feeding. Many researchers have successfully find out the functions of several proteins, using computational approach. It is in this context that in this study the structural function of melanin concentrating hormone receptor 1 through docking studies has been done to make sure that those who are working to address the problem of obesity while trying to discover the effective drugs gain much insight about this receptor. The in silico methods have been used to predict the model of melanin concentrating hormone receptor 1 . The template used for model prediction was human delta opioid receptor with the accession number $4 \mathrm{~N} 6 \mathrm{H}$. The predicted model has been evaluated and found to be of good quality. Docking was done to investigate the interaction between the ligand; a bifunctional peptide '1-oleoyl-r-glycerol' and the predicted model of melanin concentrating hormone receptor 1 which showed that fourteen residues interacted between the predicted model and ligand. Among interacting residues, it was realized that some of them are involved in sugar metabolism. Thus this study suggests a potential candidate for drug design against cancer and diabetes.
\end{abstract}

Keywords: obesity, MCHR-1, docking, structural function, 3D structure, phylogenetic analysis, interacting residues. 


\section{INTRODUCTION}

Melanin concentrating hormone receptor 1 is a G-protein coupled receptor expressed in part of the nervous system that regulates feeding and homeostasis, which are hypothalamus and zona incerta. An experiment done showed melanin concentrating hormone receptor 1 as appetite stimulation where artificial made melanin concentrating hormone receptor 1 or melanin concentrating hormone, when given to mice; increased feeding (Gibson et al. 2004). The world health organization reported obesity as a global epidemic, this disease is associated with multiple severe diseases: like diabetes mellitus, hypertension and cardiovascular diseases which clearly explain the severity of this pandemic disease (Bray and Bellanger 2006).

The induction of obesity is due to dietary contents. High-fat diets are often used to differentiate phenotype of genetically manipulated mice from their wild type control. It has been shown that when knockout mice are fed the dietary rich in fat contents, weight gain was lower compared to the wild type (Chen et al. 2015)

While searching treatments for obesity, researchers have discovered different antagonists for this MCHR1 like 181 quinoline/quinazoline derivatives that are the most potential MCHR1 antagonists found to successfully bind MCHR1(Wu et al. 2014).The binding of MCH to MCHR1 induces obesity. Fortunately, it has been demonstrated that some small molecules are capable to inhibit this interaction. For instance, bifunctional ligand for inhibiting tight-binding protein-protein interactions (Ivan et al. 2016). Within this angle falls 1-Oleoyl-R-glycerol ligand or called $(2 R)$ 2,3-dihydroxypropyl (9Z)-octadec-9-enoate (Kim et al. 2019). The main role of bifunctional ligand is to inhibit the building of $\mathrm{MCH}$ by activating its receptor, resulting in obesity. Docking MCHR1 with 1-Oleoyl-R-glycerol ligand would lead to its inactivation, thence the alleviation of obesity and its associated diseases.

Many pharmaceutical companies have also shown their intervention but they are unable to develop an efficient compounds that demonstrates the efficacy of anti-obesity for clinical trial although there has been also noted lack of efficacy in pharmacodynamics properties to test the hypothesis (Macneil 2015). Failure to detect the anti-obesity efficacy with MCHR1 antagonists in the clinics is associated with different reasons like disconnection between rodent and human studies. Although the study done for the first time in the laboratory might not have the property to sufficiently block MCHR1 so that it could achieve the energy balance, there had been no any study measuring the level of the receptor occupancy for the clinical compounds (Macneil 2015).

Several studies are being conducted to find a cure against obesity and it is undoubtedly the reason why this work on the structural function of melanin concentrating hormone receptor 1 through docking studies, has been conducted to at least provide scientific insights to medicine developers if they are to come up with effective anti-obesity drug. 


\section{MATERIAL AND METHODS}

Molecular modelling, three dimension structure model prediction and molecular docking techniques, were used in this research. The sequence of melanin concentrating hormone receptor 1(Accession number: Q99705) was downloaded from the UniProt database (Apweiler et al. 2004). The search for homologous proteins was done using BLAST by submitting the FASTA sequence of MCHR1 to BLAST, where PSI-BLAST (Altschul et al. 1997) tool was activated with PDB as database.

Multiple sequence alignment was carried out by accessing CLUSTAL omega (Sievers et al. 2011). Through this program, 5 subjects were aligned onto the query sequence. The phylogenetic analysis was done to find the evolutionary history of the query sequence and its fifteen homologous proteins aligned to the sequence of interest. PHYLIP package was used to find out the consensus tree (Liu and Beckenbach 1992), various programs of the package namely SEQBOOT, PROML and CONSENSE, were used in this regards.

Three dimension (3D) structure prediction was done with the help of SWISS-MODEL server workspace (Liu and Beckenbach 1992) (Arnold et al. 2006). To assess the predicted model, coordinates of the predicted model were subjected to different web servers: ERRAT web server (Kiefer et al. 2009), to control the quality factor of the predicted model were submitted to PROCHECK server (Karolina A. Majorek, Matthew D. Zimmerman, Marek Grabowski, Ivan G. Shabalin, Heping Zheng 2020), program of the SWISS- MODEL to check stereochemistry features of the model (Peitsch 1997).

Docking studies were performed by opening the energy minimized 3D model of melanin concentrating hormone receptor 1 and the 3D structure of (2R)-2, 3-dihydroxypropyl (9Z)octadec-9-enoate into HEX software. This has been done by first loading the shape and electrostatic energy of the software. After docking process, both the interactions and the conformation energies were written.

\section{RESULTS AND DISCUSSION}

\subsection{Molecular Modeling}

Sequence homologs of melanin concentrating hormone receptor 1 (accession number: Q99705) were obtained from pdb databank using PSI-BLAST, which helped to identify homologous proteins having higher sequence identity (Figure-1). 


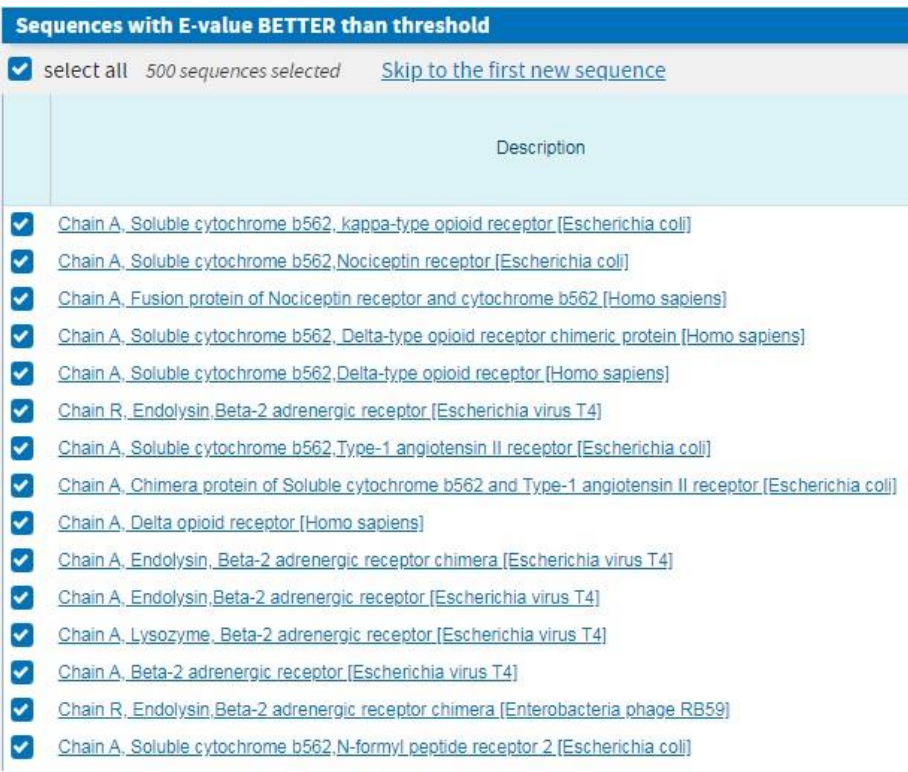

PSI-BLAST iteration 21

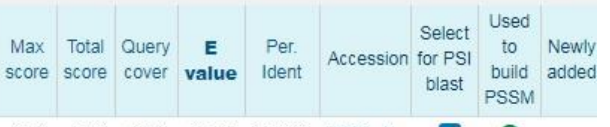

$292 \quad 292 \quad 93 \% \quad 8 \mathrm{e}-95 \quad 22.86 \% \quad 6 \mathrm{~B} 73 \quad \mathrm{~A}$

$284 \quad 284 \quad 92 \% \quad 7 e-92 \quad 23.92 \% \quad 5$ DHG A

$284 \quad 284 \quad 92 \% \quad 1 \mathrm{e}-91 \quad 23.92 \% \quad 4$ EA3 A

$\begin{array}{llllll}282 & 282 & 91 \% & 2 \mathrm{e}-91 & 23.33 \% & 4 \mathrm{~N} 6 \mathrm{H} \mathrm{A}\end{array}$

$282 \quad 282 \quad 91 \% \quad 4 e-91 \quad 23.33 \% \quad$ 4RWA A

$\begin{array}{llllll}277 & 277 & 91 \% & 8 \mathrm{e}-88 & 16.99 \% & \text { 3SN6 R }\end{array}$

$\begin{array}{llllll}272 & 272 & 91 \% & 2 \mathrm{e}-87 & 22.34 \% & 4 \text { YYA A }\end{array}$

$270 \quad 270 \quad 90 \% \quad 1$ e- $86 \quad 22.25 \% \quad 4 Z \cup D$ A

$271 \quad 271 \quad 91 \% \quad 2 \mathrm{e}-86 \quad 22.31 \% \quad 6$ PT2 $\mathrm{A}$

$\begin{array}{llllll}272 & 272 & 91 \% & 2 \mathrm{e}-86 & 17.88 \% & 6 \mathrm{MXT} \text { A }\end{array}$

$268 \quad 268 \quad 87 \% \quad 6 \mathrm{e}-85 \quad 17.89 \% \quad 5 \mathrm{JQH} A$

$264 \quad 264 \quad 87 \% \quad 3 e-83 \quad 17.62 \% \quad 4$ LLE A

$261 \quad 261 \quad 87 \% \quad 3 e-82 \quad 17.62 \% \quad 40 K X$ A

$257 \quad 257 \quad 85 \% \quad 4 \mathrm{e}-80 \quad 16.80 \% \quad 6 \mathrm{~N} 13 \mathrm{R}$

$252 \quad 252 \quad 91 \% \quad 2 \mathrm{e}-79 \quad 21.57 \% \quad 6$ LW5 A

$\begin{array}{ll}\nabla & 0 \\ 0 & 0 \\ 0 & 0 \\ 0 & 0 \\ 0 & 0 \\ 0 & 0 \\ 0 & 0 \\ 0 & 0 \\ 0 & 0 \\ 0 & 0 \\ 0 & 0 \\ 0 & 0 \\ 0 & 0 \\ 0 & 0 \\ 0 & 0\end{array}$

Figure 1 List of top fifteen homologous proteins retrieved after 21st iteration by PSI-BLAST

Findings of this data are in line with published data, in which PSI-BLAST was applied to study sequence similarity in protein (Altschul et al. 1997), (Aravind and Koonin 1999) (Hu and Kurgan 2019).

Multiple sequence alignment performed successfully by CLUSTAL Omega was able to display some conserved residues between MCHR1and top five homologous proteins retrieved from PDB databank by PSI-BLAST (Figure-2 ).

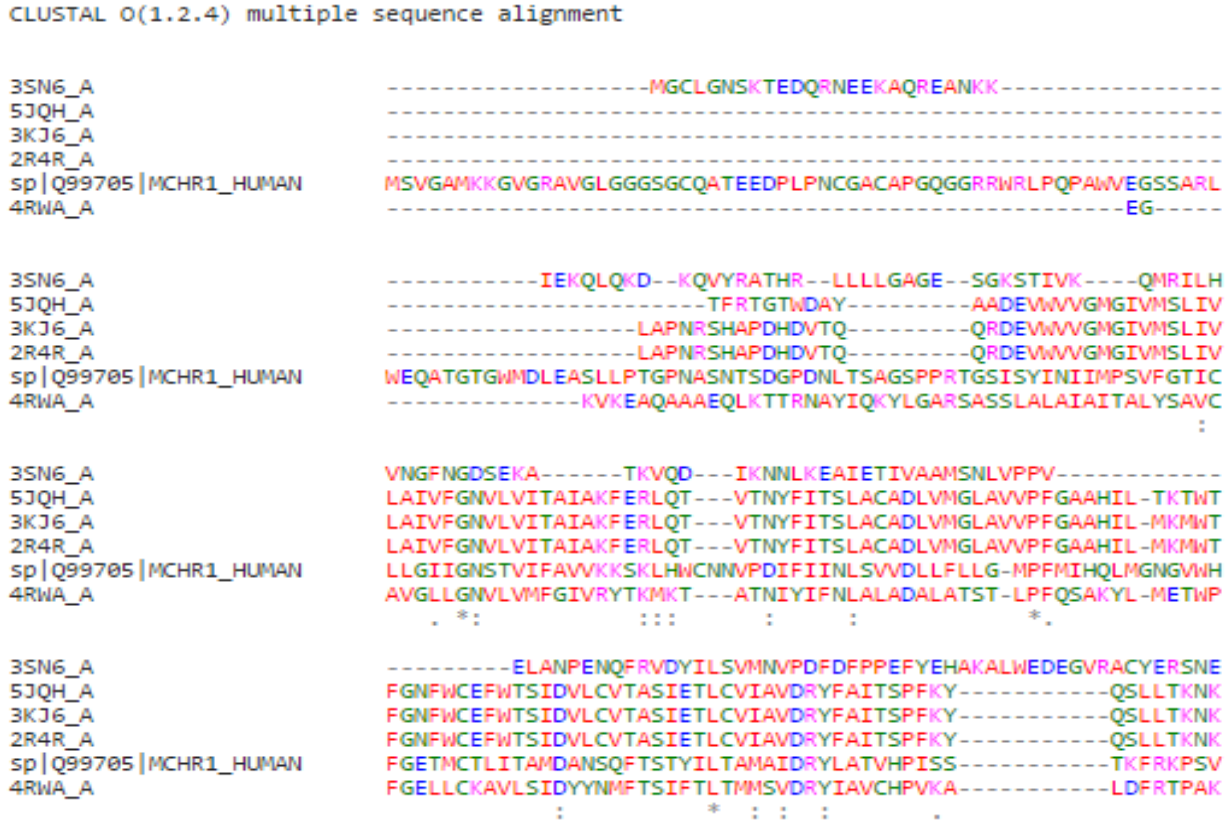
: 

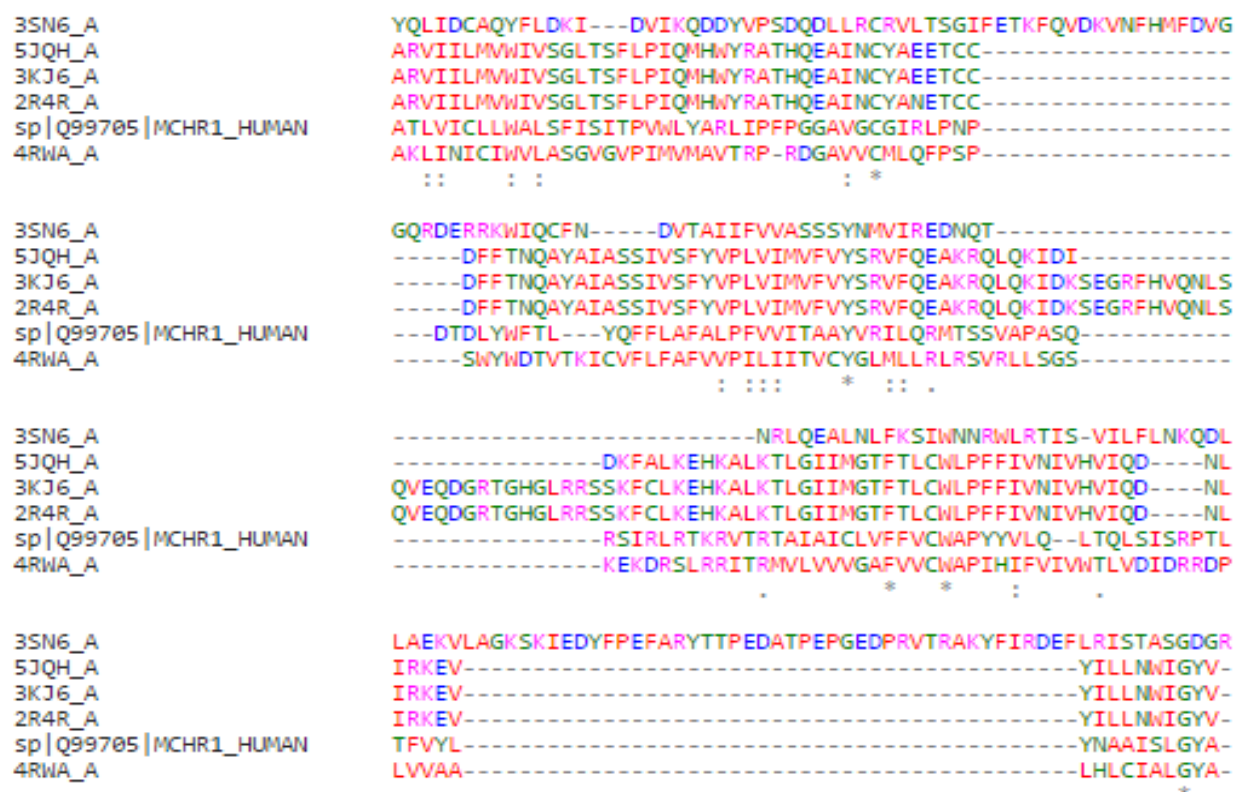

Figure 2 Multiple sequence alignment of top five homologous proteins resulted from sequence alignment

The above figure shows some conserved residues like alanine which is involved in the sugar metabolism and its deficiency is seen in case like hypoglycemia, elevated insulin and glucagon levels (Shi et al. 2002).

PHYLIP package was used to construct the consensus tree to check the level of relationship among compared homologous proteins (Figure-3); there are MCHR1 and its homologous proteins. 


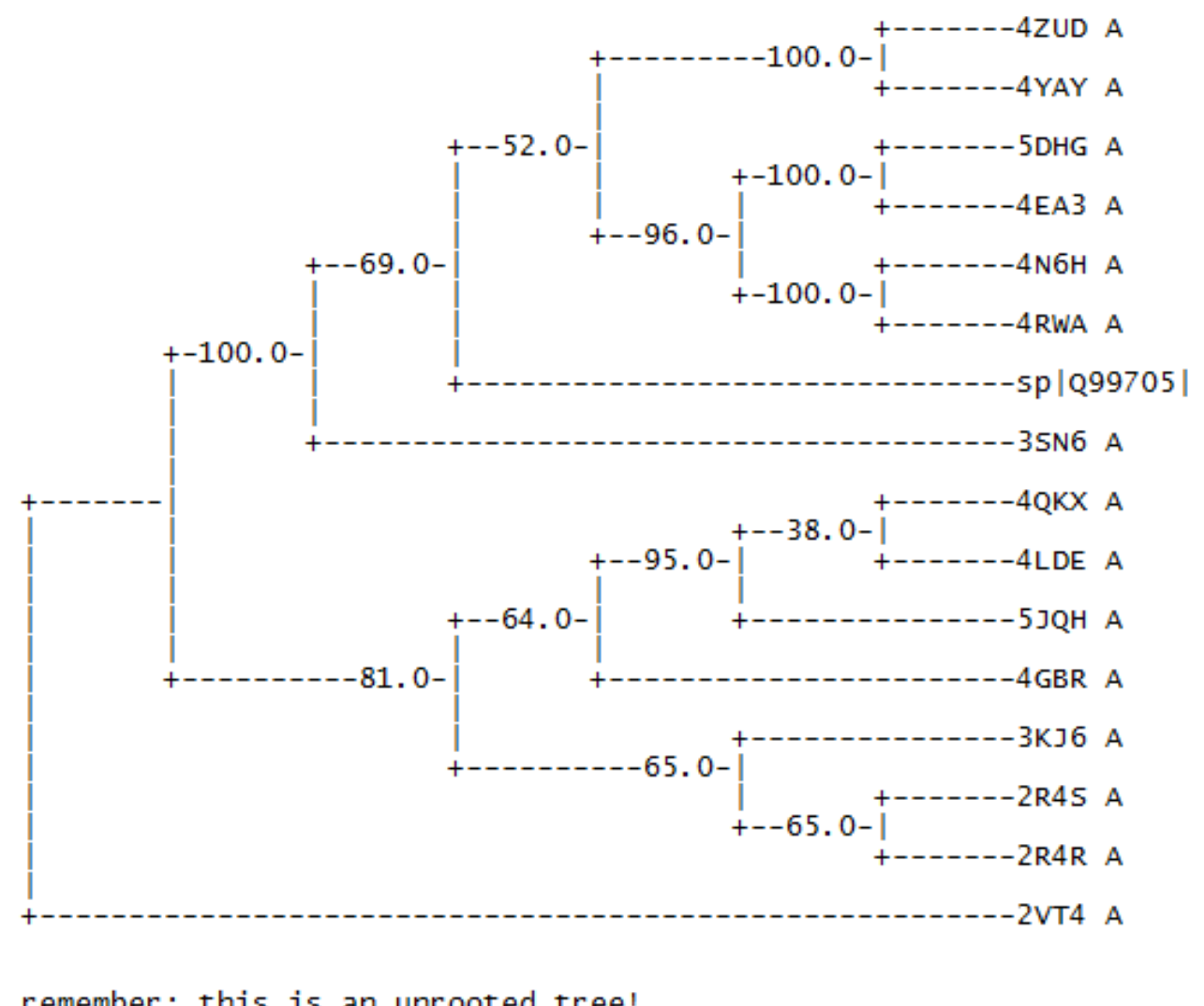

remember: this is an unrooted tree!

Figure 3 Consensus tree generated for MCHR1 using PROML as program of PHYLIP package

It is seen on the consensus tree that "4RWA and 4N6H", "4ZUD and 4YAY", "5DHG and 4EA3" are sister clades due to the fact of being on the same tree branch while both 3SN6 and 2VT4 are considered as a precursor and out group of MCHR1; respectively. Since the above couples of sister clades occur, maximum times in 100 times; anyone can be used as a template for homology modeling purposes. It is in this light that $4 \mathrm{~N} 6 \mathrm{H}$ was selected to be used as a template in predicting the model structure of melanin concentrating hormone receptor 1 through comparative modeling. The phylogenetic analysis enabled to check the subject which is more related to the query in terms of homology because an accurate prediction must be based on the sequence similarity or the evolution (Cavalli-Sforza and Edwards 1967). Similar philosophy was applied in a research conducted on mitochondrial genome sequence (Zhao et al. 2016), and their findings are in agreement with data discussed in this article.

\subsection{Three Dimension Structure Model Prediction}

The template protein structure $(4 \mathrm{~N} 6 \mathrm{H})$ chosen for 3D structure prediction of melanin concentrating hormone receptor 1 , was selected based on the results of sequence alignment and phylogenetic 
analysis. This protein satisfies three criteria: high sequence identity among other homologous proteins, high resolution as determined using $\mathrm{X}$-ray crystallography technique and belong to clades that are closer to the query sequence.

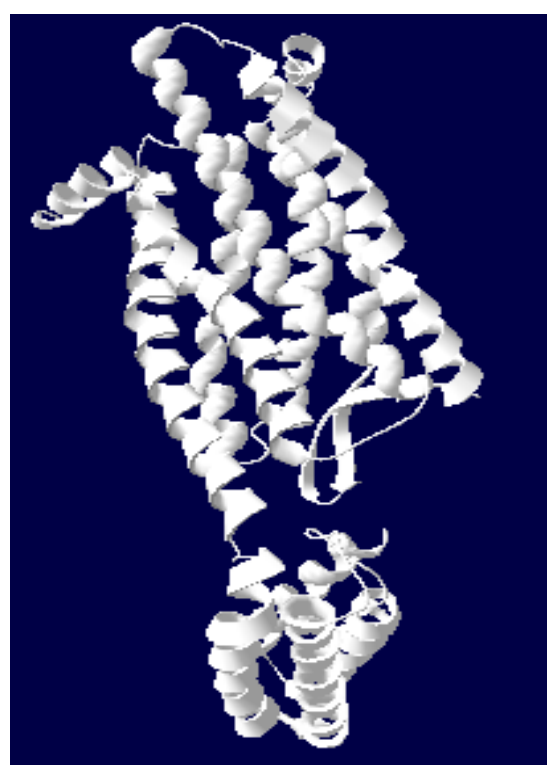

(a)

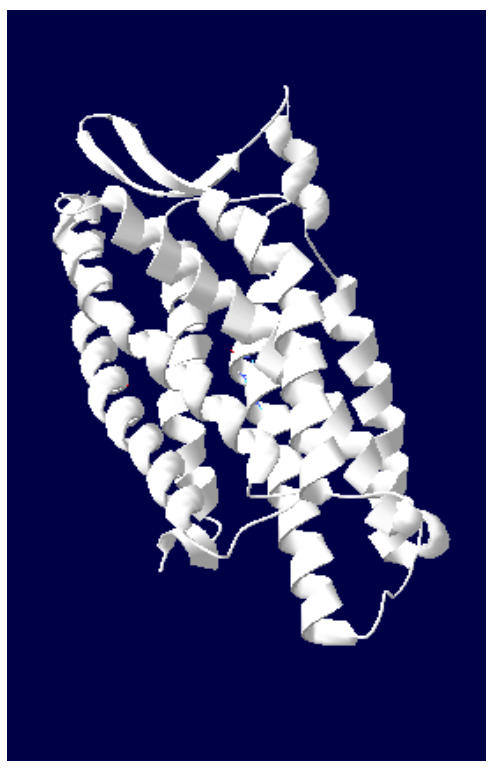

(b)

Figure 4 (a) 3D structure of the predicted model of melanin concentrating hormone receptor compared (b) X-ray structure of opioid receptor $(4 \mathrm{~N} 6 \mathrm{H})$ protein used as template during homology modeling process. Images were generated by SPDB Viewer package.

Coordinates of the initial model predicted has been assessed using ERRAT web server. Overall Quality factor of the predicted model, displayed by ERRAT server was 95.668 (Figure-5). 
Program: ERRAT2

File:/var/www/SAVES/Jobs/1187637//errat.pdb

Chain\#:1

Overall quality factor**: 95.668

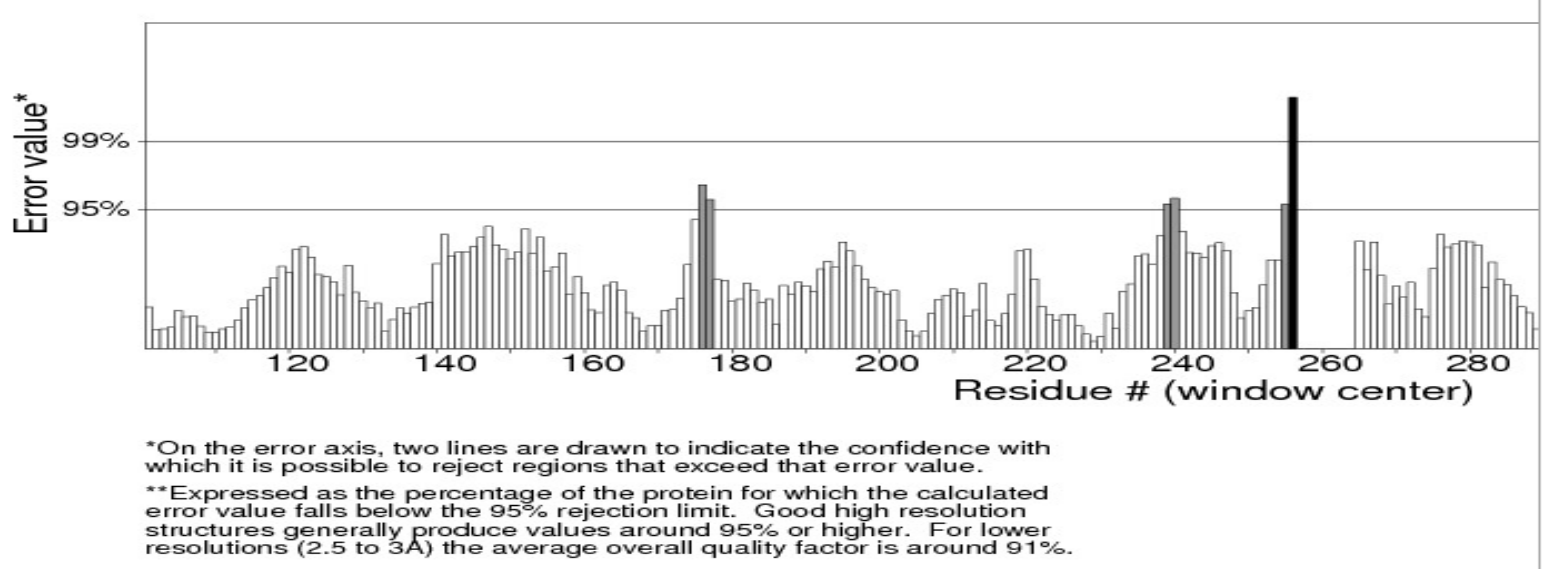

Figure 5 Quality factor of the predicted model, displayed by ERRAT server, where the overall quality factor is 95.668

The high-quality factor of the predicted model suggested that the model is of acceptable range and can be used for further analysis (Kiefer et al. 2009).

The assessment stereochemistry aspects of the model by PROCHECK server revealed how distribution of residues in regions of Ramachandran plot. 


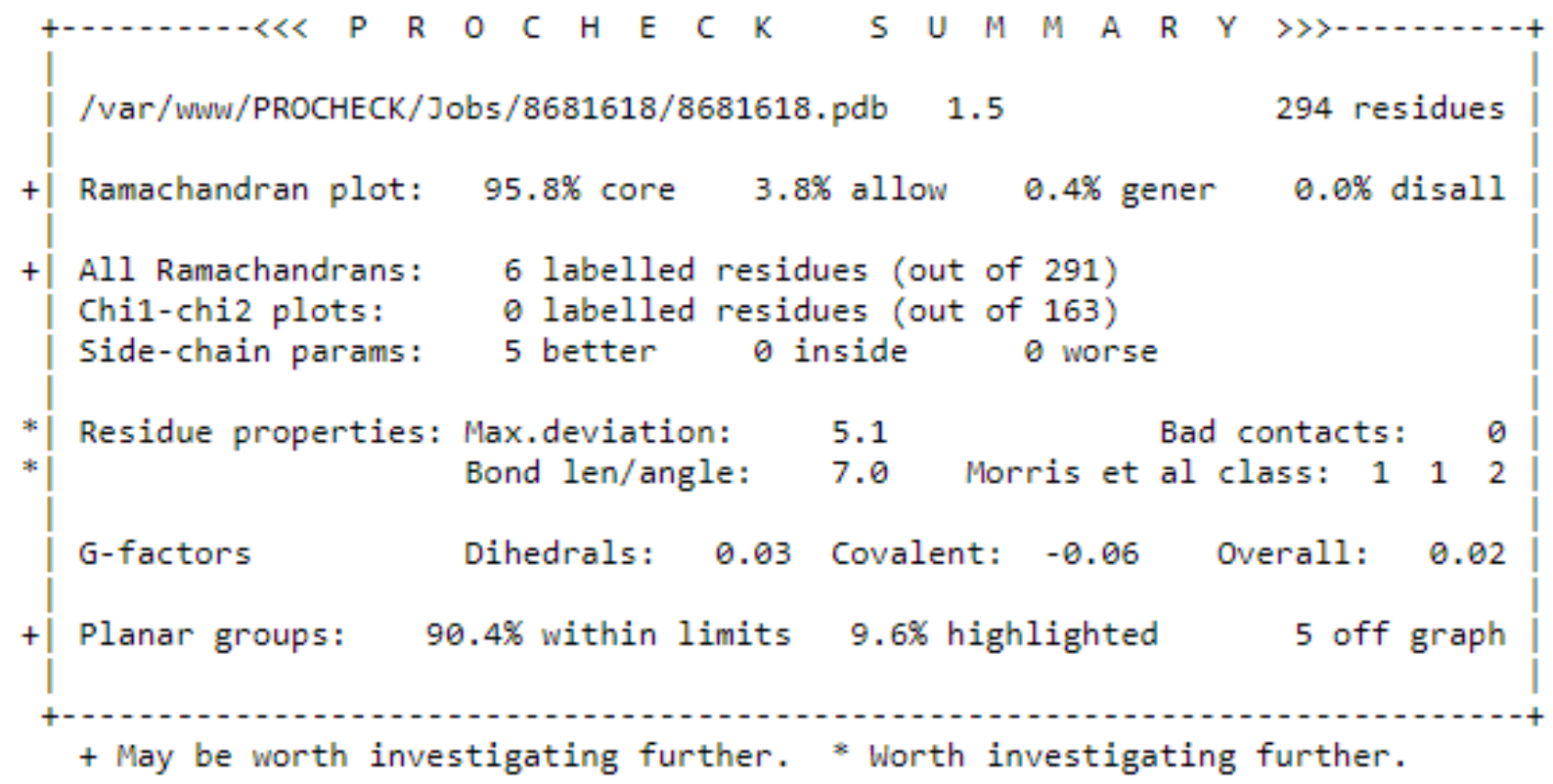

Figure 6 PROCHECK results showing stereochemistry features of the predicted model where $94.3 \%$ of the residues in the core region, the remaining $5.4 \%$ in the allowed region and $0.00 \%$ in disallowed region.

These results suggest that the predicted model was of high quality (Figure-6) as similar data were published by researchers who used ERRAT and PROCHECK to investigate the quality of predicted models (Altschul et al. 1997), (Aravind and Koonin 1999) (Hu and Kurgan 2019).

\subsection{Molecular Docking Studies}

Molecular Docking studies of the 1-Oleoyl-R-glycerol ligand onto the receptor which is the predicted 3D model of melanin concentrating hormone receptor 1, was successfully completed using HEX software. HEX helped to generate ten docked conformations. Docking energy was recorded to be $\left(-210.01 \mathrm{kcal} \mathrm{mol}^{-1}\right)$. A low (negative) energy indicates a thermostable system and thus a likely binding interaction (Cao et al. 2011). 


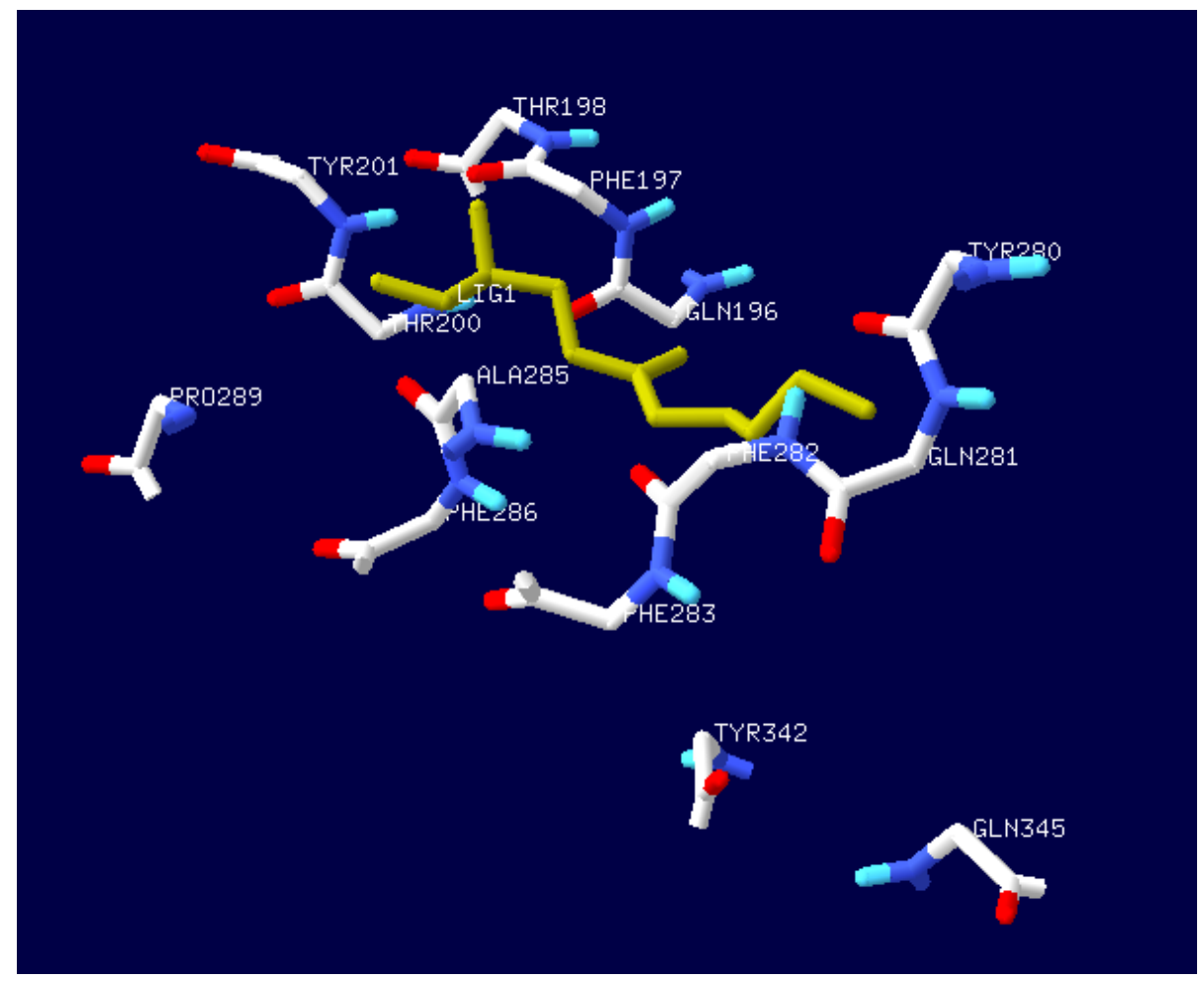

Figure 7 Interacting residues between ligand 1-Oleoyl-R-glycerol displayed in yellow color and the predicted model of melanin concentrating hormone receptor 1 used as receptor, the image was generated by SPDB Viewer package.

Fourteen residues interacted with the ligand, 1-Oleoyl-R-glycerol, as displayed using SWISS PDB

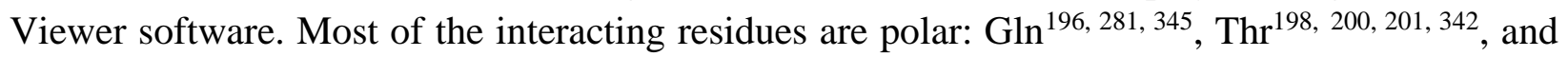
$\mathrm{Tyr}^{342}$. However, the non-polar residues are $\mathrm{Phe}^{197,} 282,283, \mathrm{Ala}^{285}$ and $\mathrm{Pro}^{289}$. No basic or acidic residue was involved in the interaction with the 1-Oleoyl-R-glycerol ligand. These interacted residues have a different role in the organism: alanine is involved in the sugar metabolism and its deficiency is seen in cases like hypoglycemia, elevated insulin and glucagon levels (Shi et al. 2002) Glutamine is involved in maintaining normal and steady blood sugar levels (Burke et al. 1989). Docking approach has been proven to give insights with regards to explain protein-protein interaction or protein-ligand interaction, at molecular level; published data (Du et al. 2016), (Burke et al. 1989), (Hu and Kurgan 2019) are in accordance with findings of this research.

\section{CONCLUSION}

Studies carried out in this research have proven that melanin concentrating hormone receptor 1 demonstrates characteristics of transmembrane proteins as its template is also a transmembrane protein. Docking studies assured the affinity of 1-Oleoyl-R-glycerol ligand towards the predicted 
model. Fourteen residues of the predicted 3D model were seen to be interacting with 1-Oleoyl-Rglycerol ligand; in other words, this study showed that melanin concentrating hormone receptor 1 has the binding site of 1-Oleoyl-R-glycerol ligand.

This study, melanin concentrating hormone receptor 1 has demonstrated the characteristics of a factor that may cause obesity since some of interacting residues between receptor-ligand, are involved in glucose metabolism. This research shades lights into molecular interaction between docked molecules, and can be referred to while designing drug for treating obesity. However, further investigation would consider the molecular dynamics simulation aspect, before starting in vitro experiments, for a complete drug design against obesity.

\section{References}

Altschul, Stephen F, Thomas L Madden, Alejandto A Schäffer, Jinghui Zhang, Zheng Zhang, Webb Miller, and David J Lipman. 1997. "Gapped BLAST and PSI-BLAST:A New Generation of Protein Database Search Programs." Nucleic Acids Res 25 (17): 3389-3402.

Apweiler, Rolf, Amos Bairoch, Cathy H Wu, Winona C Barker, Brigitte Boeckmann, Serenella Ferro, Elisabeth Gasteiger, et al. 2004. "UniProt: The Universal Protein Knowledgebase." Nucleic Acids Research 32 (Database issue): D115-9.

Aravind, L., and Eugene V. Koonin. 1999. "Gleaning Non-Trivial Structural, Functional and Evolutionary Information about Proteins by Iterative Database Searches." Journal of Molecular Biology 287 (5): 1023-40.

Arnold, Konstantin, Lorenza Bordoli, Jürgen Kopp, and Torsten Schwede. 2006. "The SWISSMODEL Workspace: A Web-Based Environment for Protein Structure Homology Modelling." Bioinformatics (Oxford, England) 22 (2): 195-201.

Bray, George A, and Tracy Bellanger. 2006. "Epidemiology, Trends, and Morbidities of Obesity and the Metabolic Syndrome." Endocrine 29 (1): 109-17.

Burke, D J, J C Alverdy, E Aoys, and G S Moss. 1989. "Glutamine-Supplemented Total Parenteral Nutrition Improves Gut Immune Function.” Archives of Surgery 124 (12): 139699.

Cao, Huijuan, Hui Hu, Ben Colagiuri, and Jianping Liu. 2011. "Medicinal Cupping Therapy in 30 Patients with Fibromyalgia: A Case Series Observation." Forschende Komplementarmedizin 18 (3): 122-26.

Cavalli-Sforza, L L, and A W F Edwards. 1967. "Phylogenetic Analysis Models and Estimation Procedures." American Journal of Human Genetics 19 (3 Pt 1): 233-57.

Chen, Yanyun, Changzhi Hu, Chiun-kang Hsu, Qing Zhang, Chen Bi, Mark Asnicar, Hansen M 
Hsiung, et al. 2015. "Targeted Disruption of the Melanin-Concentrating Hormone Receptor1 Results in Hyperphagia and Resistance to Diet-Induced Obesity" 143 (September): 246977.

Du, Xing, Yi Li, Yuan-Ling Xia, Shi-Meng Ai, Jing Liang, Peng Sang, Xing-Lai Ji, and ShuQun Liu. 2016. "Insights into Protein-Ligand Interactions: Mechanisms, Models, and Methods." International Journal of Molecular Sciences 17 (2): 144.

Gibson, William T, Pavlos Pissios, Daniel J Trombly, Jian Luan, Julia Keogh, Nicholas J Wareham, Eleftheria Maratos-flier, et al. 2004. "Melanin-Concentrating Hormone Receptor Mutations and Human Obesity: Functional Analysis" 12 (5): 743-49.

Hu, Gang, and Lukasz Kurgan. 2019. "Sequence Similarity Searching." Current Protocols in Protein Science 95 (1): 1-19.

Ivan, Taavi, Erki Enkvist, Birgit Viira, Ganesh Manoharan, Gerda Raidaru, Alexander Pflug, Kazi Asraful Alam, Manuela Zaccolo, Richard A Engh, and Asko Uri. 2016. "Bifunctional Ligands for Inhibition of Tight- Binding Protein-Protein Interactions." Bioconjugate Chem, ACS Publications 27: 1900-1910.

Karolina A. Majorek, Matthew D. Zimmerman, Marek Grabowski, Ivan G. Shabalin, Heping Zheng, Wladek Minor. 2020. "Assessment of Crystallographic Structure Quality and Protein-Ligand Complex Structure Validation.” Structural Biology in Drug Discovery, 253-75.

Kiefer, Florian, Konstantin Arnold, Michael Künzli, Lorenza Bordoli, and Torsten Schwede. 2009. "The SWISS-MODEL Repository and Associated Resources." Nucleic Acids Research 37 (SUPPL. 1): 387-92.

Kim, Sunghwan, Jie Chen, Tiejun Cheng, Asta Gindulyte, Jia He, Siqian He, Qingliang Li, et al. 2019. "PubChem 2019 Update: Improved Access to Chemical Data." Nucleic Acids Research 47 (D1): D1102-9.

Liu, Hong, and Andrew T. Beckenbach. 1992. "Evolution of the Mitochondrial Cytochrome Oxidase II Gene among 10 Orders of Insects." Molecular Phylogenetics and Evolution 1 (1): 41-52.

Macneil, Douglas J. 2015. "The Role of Melanin-Concentrating Hormone and Its Receptors in Energy Homeostasis" 4 (April 2013): 1-14. https://doi.org/10.3389/fendo.2013.00049.

Peitsch, Manuel C. 1997. "SWISS-MODEL and the Swiss-PdbViewer : An Environment for Comparative Protein Modeling," 2714-23.

Shi, Zhengshuang, C. Anders Olson, George D. Rose, Robert L. Baldwin, and Neville R. Kallenbach. 2002. "Polyproline II Structure in a Sequence of Seven Alanine Residues." Proceedings of the National Academy of Sciences of the United States of America 99 (14): 9190-95.

Sievers, Fabian, Andreas Wilm, David Dineen, Toby J Gibson, Kevin Karplus, Weizhong Li, 
Rodrigo Lopez, et al. 2011. "Fast, Scalable Generation of High-Quality Protein Multiple Sequence Alignments Using Clustal Omega." Molecular Systems Biology 7 (1): 539.

Wu, Mingwei, Yan Li, Xinmei Fu, Jinghui Wang, Shuwei Zhang, and Ling Yang. 2014. "Profiling the Interaction Mechanism of Quinoline / Quinazoline Derivatives as MCHR1 Antagonists : An in Silico Method," no. Md: 15475-502.

Zhao, Y. Y., L. N. Su, Z. M. Zhang, and X. Y. Wang. 2016. "Phylogenetic Relationships of Pseudohynobius (Urodela, Hynobiidae) Inferred from DNA Barcoding Analysis." Genetics and Molecular Research 15 (2): 1-7. 